\title{
Analysis of Vorticity Budget for a Developing Extraordinary Arctic Cyclone in August 2016
}

\author{
Ryota Ishiyama ${ }^{1}$ and Hiroshi L. Tanaka ${ }^{1,2}$ \\ ${ }^{1}$ Graduate School of Life and Environmental Sciences, University of Tsukuba, Ibaraki, Japan \\ ${ }^{2}$ Center for Computational Sciences, University of Tsukuba, Ibaraki, Japan
}

\begin{abstract}
In this study, we conducted a domain-integrated vorticity budget analysis to quantitatively understand the developing mechanism of the Arctic Cyclone (AC) in August 2016 (AC16). The results showed that the vorticity enhancement of the AC16 was dominated by the horizontal flux convergence of vorticity at all layers with a maximum near the tropopause. The enhancement near the tropopause was characterized not only by the horizontal supply but also by the vertical transport of vorticity. In the boundary layer within the AC16, the convergence of horizontal winds and the corresponding divergence of vertical winds occurred. In addition, during the merging process, updrafts were dominant in the troposphere due to the structure of the mid-latitude cyclone. These structures caused the upward transport of vorticity to the tropopause, which is considered as an important internal process of the AC16. However, time-averaged vorticity budget during the developing stage indicated that the vertical flux term and the divergence term compensate with each other. As a result, it was concluded that the $\mathrm{AC}$ is excited and maintained by the merging of the vortices associated with the migrating mid-latitude cyclone and polar vortex.
\end{abstract}

(Citation: Ishiyama, R., and H. L. Tanaka, 2021: Analysis of vorticity budget for a developing extraordinary arctic cyclone in August 2016. SOLA, 17, 120-124, doi:10.2151/sola.2021-020.)

\section{Introduction}

In recent years, Arctic warming is known to be faster than the global average (e.g., Zhang 2005), which is referred to as Arctic amplification (e.g., Serreze and Francis 2006; Cohen et al. 2014; Screen et al. 2018). In the rapidly changing environment, there are many studies about the relationship between sea ice melting and cyclones in the Arctic (e.g., Simmonds and Keay 2009; Koyama et al. 2017). Analyzing the developing mechanism of the cyclones in the Arctic is important for understanding the recent rapid changes of the Arctic climate system and predicting these future changes.

A long-lived cyclone that develops in the Arctic, especially in summer, is called an Arctic Cyclone (AC) (Tanaka et al. 2012). Overall, the ACs have a barotropic structure of vorticity, with warm and cold cores in the lower stratosphere and the troposphere, respectively (Aizawa and Tanaka 2016). The developing mechanism of the ACs has been discussed in some previous studies; Crawford and Serreze (2016) showed that the baroclinicity over the Arctic frontal zone affected on an intensification of the ACs. Aizawa et al. (2014) indicated that the rapid development of the ACs is caused by merging with the other cyclone, which leads to the intensification of the warm core at the upper level and the enhancement of vortices at the lower level. Similar results are shown by some studies (Simmonds and Rudeva 2012, 2014; Yamagami et al. 2017). However, this mechanism remains qualitative, and it is still a challenge to quantitatively evaluate how much of the energy and vorticity in the $\mathrm{AC}$ is reliant on the other cyclone during the merging process.

Corresponding author: Hiroshi L. Tanaka, Center for Computational Sciences, University of Tsukuba, 1-1-1 Tennodai, Tsukuba, Ibaraki 305-8577, Japan. E-mail: tanaka@ccs.tsukuba.ac.jp.
A vorticity budget analysis is a standard method to quantitatively evaluate the developing mechanism of cyclones by using relative vorticity as an indicator of their development. For example, Grotjahn (1996) evaluated the predominant vorticity equation terms in a composited mid-latitude cyclone. Raymond and Carrillo (2011) examined vorticity budget of a tropical cyclone Nuri and showed that vorticity convergence dominated all other circulation tendency terms in the planetary boundary layer. In addition to the above studies, vorticity budget analysis has also been conducted for other disturbances such as a mesoscale convective vortex (Knievel and Johnson 2003; Chen and Zheng 2004) and a Japan Sea polar airmass convergence zone (JPCZ; Shinoda et al. 2020). In contrast, the evaluation of the developing dynamical processes of ACs is still unclear.

In this study, an extraordinary AC appeared in August 2016 (Yamagami et al. 2017, 2018, 2019) is selected as the target of analysis to conduct the vorticity budget. The purpose of this study is to quantitatively evaluate the domain-integrated vorticity budget for the developing process of the $\mathrm{AC}$, especially for the merging process.

\section{Data and study method}

We used horizontal wind $(\boldsymbol{V}=(u, v))$, vertical p-velocity, sea level pressure (SLP) and boundary layer height data from ERA5 (Hersbach et al. 2020). Their horizontal and temporal resolutions are $1.25^{\circ} \times 1.25^{\circ}$ and 6 hours, respectively. For the analysis of large horizontal and time scales of an AC, these thinned out resolutions may be sufficient. To facilitate the interpretation of the results, we also used the data of the tropopause height at 6-hour intervals provided by the final operational global analysis data from the Global Forecasting System of National Centers for Environmental Prediction (NCEP FNL). Its horizontal resolution is $1.0^{\circ}$ $\times 1.0^{\circ}$. lows:

The vorticity equation in Eulerian form is expressed as fol-

$$
\begin{aligned}
\frac{\partial}{\partial t} \zeta= & -\nabla \cdot(\zeta+f) \boldsymbol{V}-\frac{\partial}{\partial p}[(\zeta+f) \omega] \\
& -(\zeta+f) \nabla \cdot \boldsymbol{V}+\vec{k} \cdot\left(\frac{\partial \boldsymbol{V}}{\partial p} \times \nabla \omega\right)+\vec{k} \cdot \nabla \times \boldsymbol{F}
\end{aligned}
$$

where $\zeta$ is relative vorticity, $\omega$ vertical p-velocity, $f$ planetary vorticity, and $\vec{k}$ a unit vector of the vertical component. To simplify the analysis, we will refer to the left-hand-side term in Eq. (1) as the local change term, and the first to fifth terms on the right-hand-side terms as the horizontal flux term, the vertical flux term, the divergence term, the tilting term, and the friction term, respectively. In this study, we evaluated the friction term by the residual balance of the equation. The friction term includes the observational and analysis errors and the unresolved source and sink of vorticity. By setting up the region of a control volume, Eq. (1) can be transformed to a domain-integrated form. The flux terms are calculated using Gaussian divergence theorem. That is, these terms are derived by calculating only the fluxes across the boundary of the control volume. The other terms are calculated by the simple integration. A flux contribution by the movement of control volume was calculated in the vorticity budget. This term 
(a) time-series of minSLP

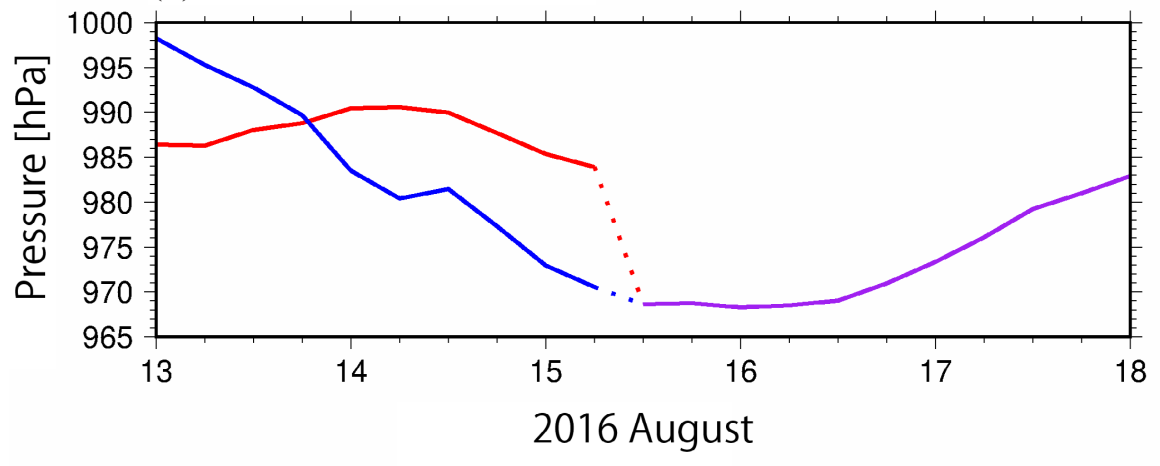

(b) $\mathrm{T}=2016081400$

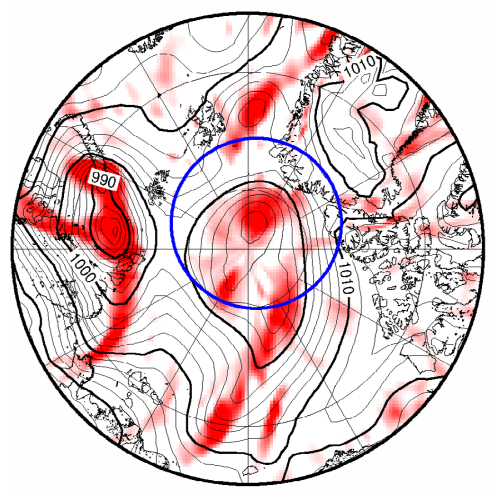

(d) $\mathrm{T}=2016081512$

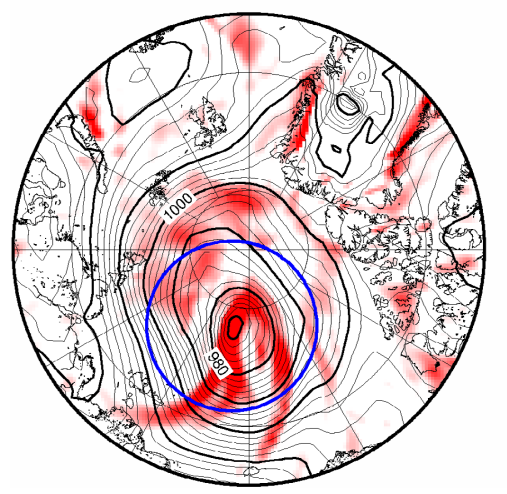

(c) $\mathrm{T}=2016081418$

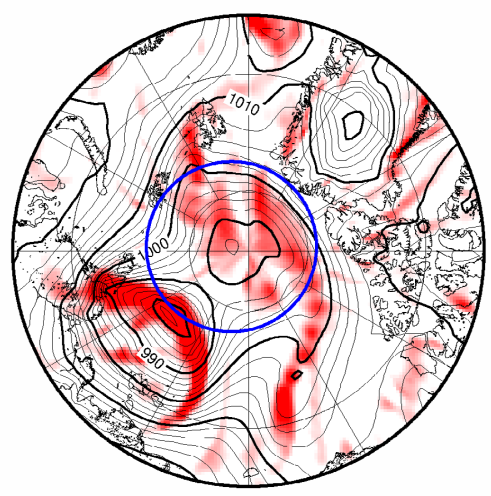

(e) $\mathrm{T}=2016081606$

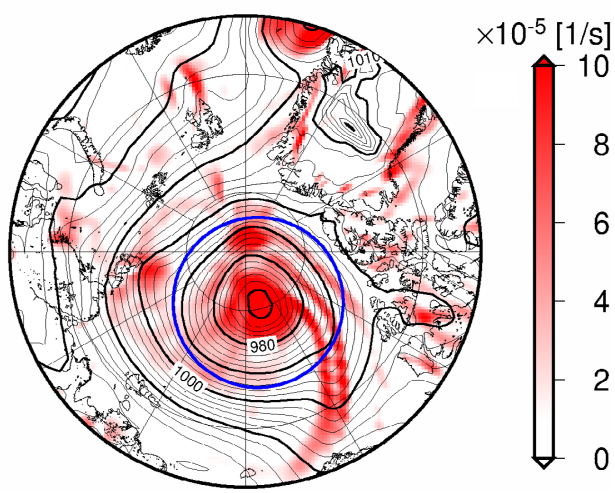

Fig. 1. (a) Time series of minimum sea level pressure (minSLP) of each cyclone: an AC16

4 (red line), a mid-latitude cyclone (blue line), and a merged AC16 (purple line). (b e) Distribution of sea level pressure (contour) and rela-

tive vorticity at $850 \mathrm{hPa}$ (shading). A blue circle represents the radius of $800 \mathrm{~km}$ from the center of the AC16, which is the boundary of the control volume for the vorticity budget analysis. was, however, disregarded since the contribution was negligibly small in most of the time.

In this study, the AC which appeared in August 2016 (hereinafter referred to as AC16) is selected as the target, because of the extraordinary strength and the long life-time for more than one month (Yamagami et al. 2017, 2018). While the analyzed cyclones characteristics vary a little according to which storm identification and tracking algorithm is used (Neu et al. 2013), we used the method developed by Aizawa and Tanaka (2016) to identify cyclone centers and minimum SLP (minSLP). Figure 1a shows the time series of minSLP of the AC16 and a merging cyclone from mid-latitudes. The peak of the AC16 was seen just after the merging with the mid-latitude cyclone. The timing of the merging of the two cyclones, as judged by the SLP, was 12UTC on 15 August. The AC16 recorded the lowest minSLP (968.3 hPa) 12 hours after the merging with the mid-latitude cyclone (00UTC on 16 August). According to Yamagami et al. (2017), the radius which was defined as the distance to the outermost closed SLP contour was more than $1,000 \mathrm{~km}$ at its peak. After that, the AC16 began to decay gradually. Figures $1 \mathrm{~b}-1 \mathrm{e}$ show the distribution of SLP and relative vorticity at $850 \mathrm{hPa}$ every 18 hour from 00UTC on 14 August. While the AC16 stayed over the Arctic Ocean at 00UTC on 14 August (Fig. 1b), a developing mid-latitude cyclone was located at Kara Sea, and it moved into the arctic region (Fig. $1 \mathrm{c})$. These two cyclones had a large vorticity and merged in the Arctic Ocean (Fig. 1d). Then, the merged cyclone showed the strongest intensification, and we can see one well-developed vortex (Fig. 1e).

The blue circles in Figs. 1b-1e indicate the location of the radius of $800 \mathrm{~km}$ from the center of the $\mathrm{AC} 16$. Considering that the $\mathrm{AC}$ has a concentric structure, we analyzed the domain-integrated vorticity budget within a radius of $800 \mathrm{~km}$ from the $\mathrm{AC}$ center as the control volume. Domain averages of the vorticity budget in Eq. (1) were calculated by the integration within this volume. The radius of $800 \mathrm{~km}$ was determined by comparing various radii. It was found that the results are not significantly different from those with a radius of $600 \mathrm{~km}$ or $1000 \mathrm{~km}$. Although the scale of the cyclones changes during their life cycle (e.g., Simmonds 2000), the control volume of the fixed radius was set in order to properly calculate the supply of vorticity from the midlatitude cyclone during the merging process. 


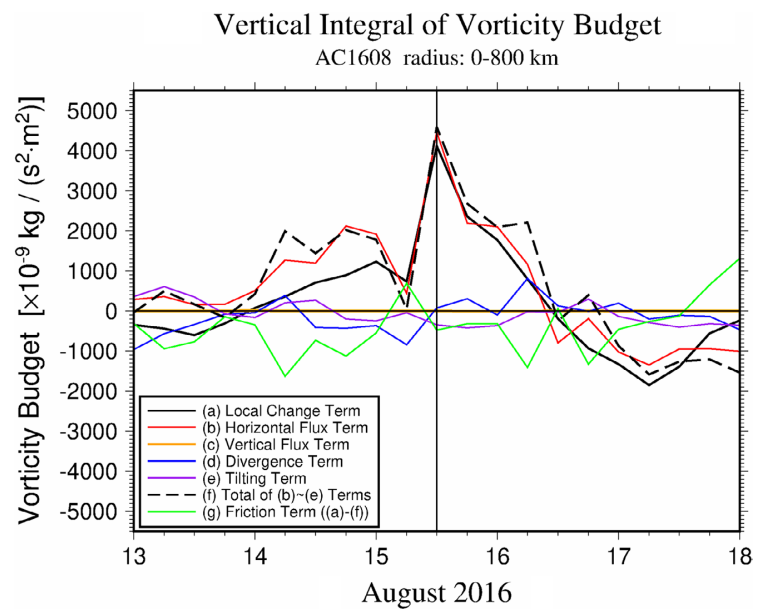

Fig. 2. Time series of the vertical integral of area averaged vorticity budget from the surface to $100 \mathrm{hPa}$. Area average is calculated within the radius of $800 \mathrm{~km}$ from the center of the AC16. The black vertical line at 12UTC on the 15th indicates the timing of the merging of the AC16 and the mid-latitude cyclone.

\section{Results and discussion}

To quantitatively evaluate how much vorticity is supplied to the AC16 from a mid-latitude cyclone during merging process, the vertical integral of vorticity budget was first examined from the surface to $100 \mathrm{hPa}$ using a boundary condition of $\omega=0$ (Fig. 2 ). The vertically integrated local change term (solid black line) increased from 00UTC on 14 August and reached its maximum at the timing of the merging (12UTC on 15 August; vertical line). The black broken line represents the sum of the right-hand side in Eq. (1), excluding the friction term. We can confirm that the summation of the right side reproduces the local change term with a good accuracy. The friction terms are generally small and negative, but the positive value shown in some periods is thought to be due to the dynamics within the resolution.

Regarding the time change of each term on the right-handside in Fig. 2, the horizontal flux term (red line) shows a similar variability to the local change term. The horizontal flux term indicates the inflow or outflow of the vorticity across the boundary of the control volume. Therefore, it was found that the vorticity increase of the AC16 during the merging process resulted from the horizontal flux convergence associated with the mid-latitude cyclone coming into the control volume. In contrast, the contributions from the vertical flux (yellow line), the divergence term (blue line) and the tilting (purple line) term were small compared with the horizontal flux term, and the friction term contributed to the decrease of vorticity. These results suggest that the vorticity generated within the control volume had little effect on the vor- ticity budget. Therefore, the present analysis of vorticity budget revealed that the vorticity merging was the most important process for the development of the AC16, which is consistent with Aizawa et al. (2014) and Yamagami et al. (2017).

In order to show the details of the internal processes of the AC16, we focus on the time change of the area-averaged vertical structure of the AC16 within the control volume. Figure 3 shows time-vertical cross sections of (a) tangential wind, (b) radial wind and (c) vertical p-velocity. The tangential and radial winds are obtained by decomposing the horizontal wind $\boldsymbol{V}=(u, v)$ at each grid point with respect to the center direction of the cyclone. During the entire 5 days, the tangential wind (Fig. 3a) shows strong positive shear with a maximum at the tropopause, indicating that the cyclonic circulation dominates. The distribution of the radial wind (Fig. 3b) is characterized by large values near the tropopause and convergence in the lowermost layer of the planetary boundary layer. This convergence in the lowermost layer is because the horizontal wind convergence occurs under the cyclone due to the surface friction within the boundary layer. The large signals near the tropopause (e.g., positive on 16 August and negative on 17 August) reflect not only the cyclone structure but also the influence of the polar vortices in the upper level. ERA5 showed that the boundary layer height within the AC16 was typically 300 1200 m (not shown). The vertical p-velocity (Fig. 3c) shows that weak downward motion dominates in the lower stratosphere. The downward motion is also dominant in the upper troposphere (e.g., 00UTC on 14 and 12UTC on 16 August). However, during the merging process, there is a pronounced upward motion throughout the troposphere (especially from 18UTC on 14 to 00UTC on 16 August). This outstanding updraft appears to reflect the structure of the mid-latitude cyclone entering the control volume.

Next, we analyzed the time changes of the vertical distribution of each term of the vorticity budget in Eq. (1). Figure 4 shows time-vertical cross sections of area-averaged vorticity budget. As in Fig. 3, they are averaged within a radius of $800 \mathrm{~km}$ from the AC center. A positive (negative) value indicates the vorticity increase (decrease). The local change term in Eq. (1) (Fig. 4a) showed that the increase of vorticity occurred at all layers during the merging process, and its maximum appears near the tropopause. The vorticity increase in the lower troposphere shows a peak at around 18UTC on 14 August. The increase of vorticity occurs first at the lower level, then it shifts to upper level. The upper-level vorticity increase shows a peak during the merging (at 12UTC on 15 August).

According to the result of the vorticity budget analysis, the horizontal flux term (Fig. 4b) indicates a similar distribution and magnitude to the local change term. Figure $4 \mathrm{~b}$ suggests that the merging of the vortex with the mid-latitude cyclone occurred first in the lower layer. As shown in Fig. 1c, the early vorticity enhancement in the lower levels is explained by the inflow of the mid-latitude vorticity into the control volume around 18UTC on the 14th. However, the peak of the vorticity enhancement near the tropopause may be influenced by the polar vortex associated with the mid-latitude cyclone, because mid-latitude cyclones do (a) Tangential Wind

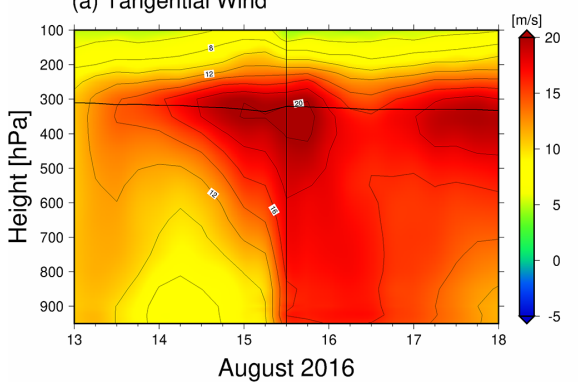

(b) Radial Wind

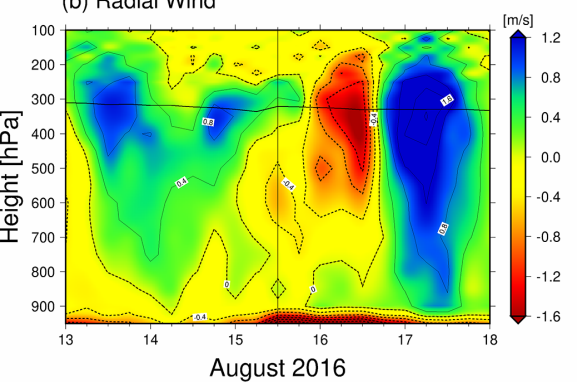

(c) Vertical P-Velocity

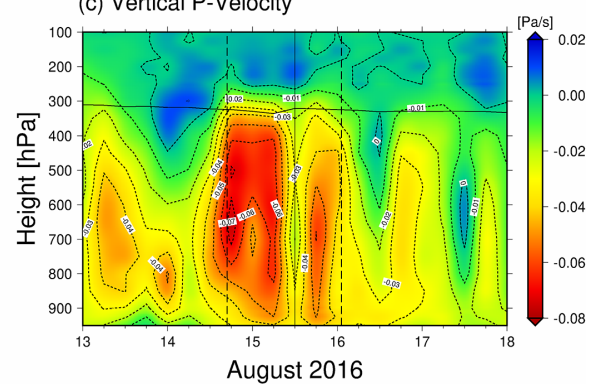

Fig. 3. Time-vertical cross sections of area-averaged (a) tangential wind, (b) radial wind, and (c) vertical p-velocity. Area average is calculated within the radius of $800 \mathrm{~km}$ from the center of the AC16. The signs of the tangential and radial winds are positive when winds blow counterclockwise and outward from the center of the AC16, respectively. The solid black line at near $300 \mathrm{hPa}$ is the tropopause height. 
(a) Local Change Term

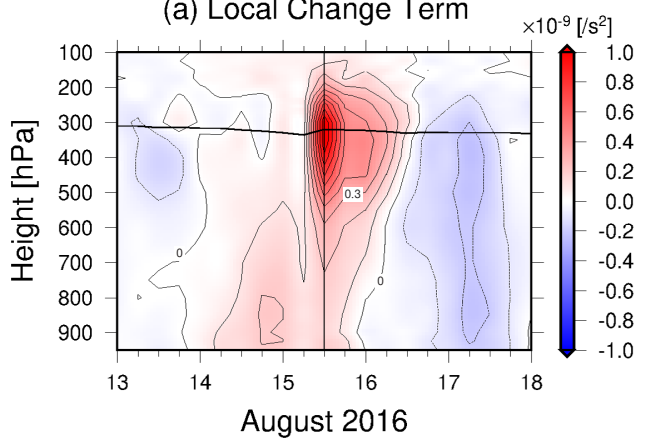

(c) Vertical Flux Term

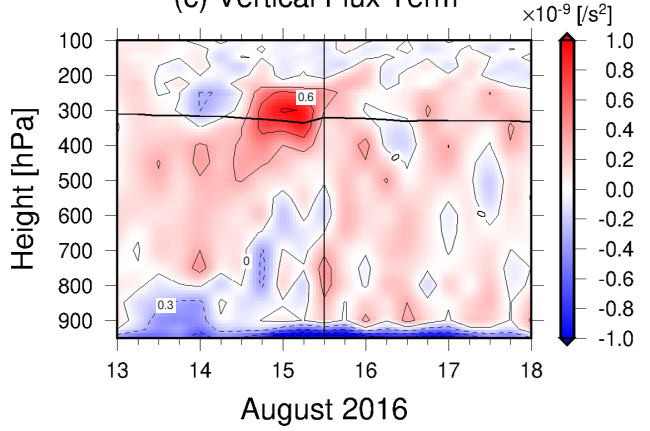

(e) Tilting Term

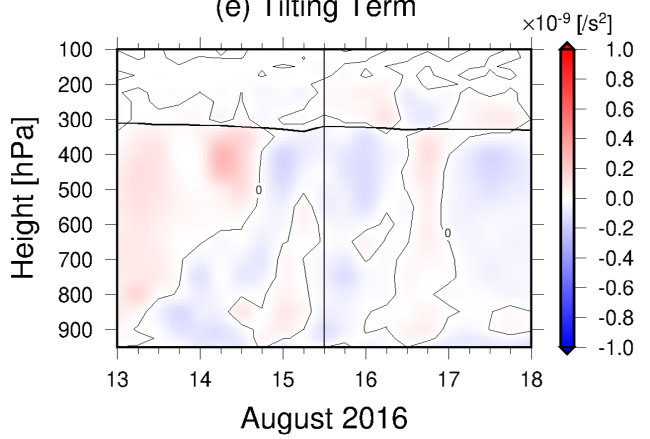

(b) Horizontal Flux Term

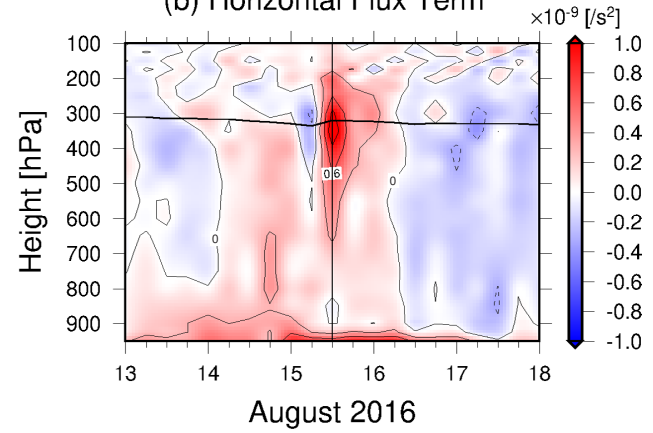

(d) Divergence Term

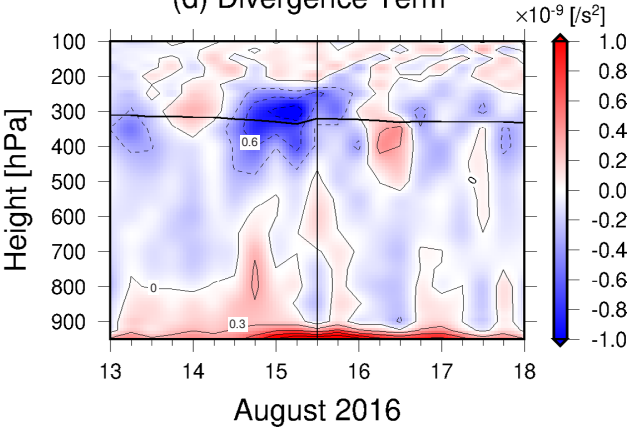

(f) Sum of (b)-(e) terms

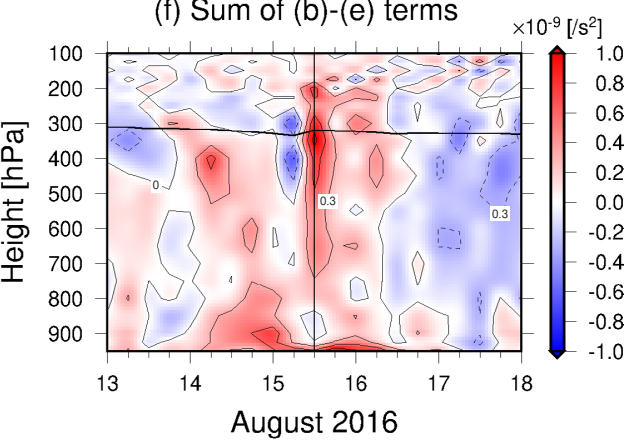

Fig. 4. Time-vertical cross sections of area-averaged (a) local change term, (b) horizontal flux term, (c) vertical flux term, (d) divergence term, (e) tilting term, and (f) the sum of the (b)-(e) terms in the vorticity equation (Eq. 1).

not always show such a large vorticity in the upper levels. The vertical flux term (Fig. 4c) and divergence term (Fig. 4d) showed generally opposite signs with large values near the tropopause and at the lowest level, which are comparable to the magnitude of the horizontal flux term. Within the boundary layer under the cyclones, the convergence of horizontal winds and the corresponding upward motions occur (Figs. $3 \mathrm{~b}$ and $3 \mathrm{c}$ ). These structures may have caused the positive contributions of the horizontal flux term and the divergence term and negative contributions of the vertical flux term. In the merging process, it was found that the air masses with large vorticity are transported to the tropopause by the upward motions in the entire troposphere (Figs. 3c and 4c). Therefore, the positive vertical flux term and the negative divergence term near the tropopause indicate the vertical convergence and horizontal divergence of the flow. The contribution from the tilting term to the vorticity budget was small in the AC16 (Fig. 4e). The distribution of the sum of the right-hand side excluding the friction term (Fig. 4f) was similar to that of the horizontal flux term (Fig. 4b), suggesting that the vertical flux and divergence terms canceled with each other. The distribution of the friction term was mostly negative especially in the lowest layer (not shown).

Finally, we analyze quantitatively which term is the most important internal process for the AC16. Figure 5 shows the time-averaged height distributions of the vorticity budget. The time average was computed for the period of strong updrafts throughout the troposphere from 18UTC on 14 to 00UTC on 16 August (see Fig. 3c). The local change term (solid black line) shows that the vorticity enhancement is found in all layers with a maximum near the tropopause. The distribution is similar to that of the horizontal flux term (red line) above $900 \mathrm{hPa}$. The vertical flux term (orange line) and divergence term (blue line) show large values at the lowest layer and near the tropopause with opposite signs, which may be caused by convergence in the boundary layer and divergence near the tropopause (as described in Fig. 4). The results suggest that the vorticity enhancement due to the vertical flux term near the tropopause is larger than that of the horizontal flux term and the combination of these terms may have strengthen the cyclonic vorticity showing the barotropic structure.

\section{Concluding summary}

In this study, a domain-integrated vorticity budget analysis was conducted for the well-developed AC16 in order to quantitatively assess the developing mechanism. Considering the concentric structure of the $\mathrm{AC}$, the control volume for the vorticity budget was determined as a circle or a cylinder with a radius of $800 \mathrm{~km}$ from the center of the AC16. We confirmed that the sum of the right-hand side of the vorticity equation Eq. (1) excluding the friction term almost coincides with the local change term, 


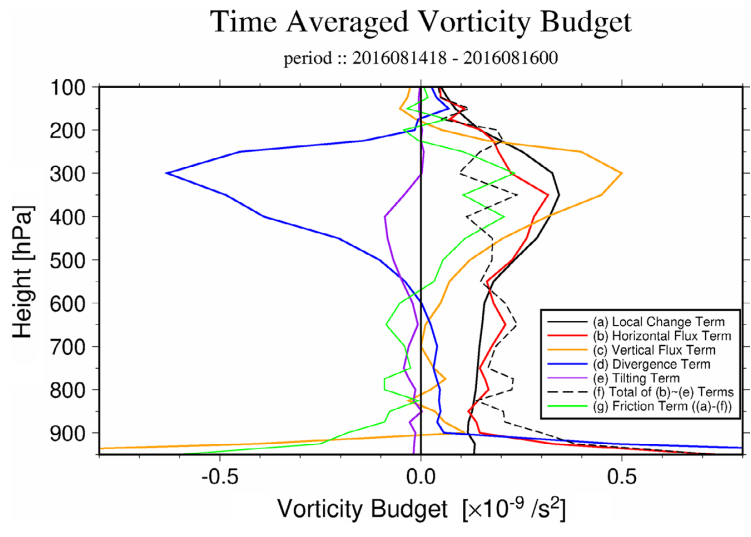

Fig. 5. Time-averaged height distribution of the vorticity budget during the development. The period is from 18UTC on 14 to 00UTC on 16 August, when the upward motion is dominant (see Fig. 3c). The vorticity budget at each height was averaged within a radius of $800 \mathrm{~km}$ from the center of the AC16.

indicating that the accuracy of the analysis was sufficiently good.

The most important result is that the development of the AC16 was caused by the horizontal flux supply of vorticity at all layers. The vertically integrated vorticity budget and the time-averaged budget during development were dominated by the horizontal flux term. The results indicate that the developing mechanism of ACs is quite different from that of the tropical and extra-tropical cyclones which have been discussed in many previous studies. The horizontal flux supply of vorticity, especially during the merging process, suggests the supply from the mid-latitude cyclone, revealing that the merging is important for the development of the AC. This is in agreement with the studies by Aizawa et al. (2014) and Yamagami et al. (2017). Although Aizawa et al. (2014) pointed out that the vertical coupling of vortices between the lower layer mid-latitude cyclone and the upper layer polar vortex is important for the development of the AC, the vorticity enhancement due to the horizontal supply occurred at all layers as found in this study.

The vorticity budget suggests that the divergence term and vertical flux term compensate with each other in the vorticity change. The divergence term showed vorticity convergence in the boundary layer and divergence near the tropopause, but these canceled each other when vertically integrated. As a result, these terms did not contribute to the vorticity enhancement in the vertically integrated budget. However, the upward transport of vorticity appeared to be an important internal process. The combination of horizontal supply and the upward transport of vorticity was considered to be important for the vorticity enhancement near the tropopause, which contributed to the strengthening of the barotropic structure of vorticity.

Although this study clarified the importance of the horizontal supply of vorticity in the development of the AC16, it suggested the need to separate the effects of mid-latitude cyclones and polar vortices on the supply of vorticity near the tropopause. Furthermore, a discussion on the role of moisture flux convergence and cloud formation for the development will also be a future work.

\section{Acknowledgements}

The authors are grateful to Prof. Mio Matsueda and Dr. Akio Yamagami of University of Tsukuba for supporting our research. This research was conducted as a part of the joint works of the general circulation laboratory at the Center for Computational Sciences of the University of Tsukuba.

Edited by: C. Kobayashi

\section{References}

Aizawa, T., and H. L. Tanaka, 2016: Axisymmetric structure of the long lasting summer Arctic cyclones. Polar Sci., 10, 192-198.

Aizawa, T., H. L. Tanaka, and M. Satoh, 2014: Rapid development of arctic cyclone in June 2008 simulated by the cloud resolving global model NICAM. Meteor. Atmos. Phys., 126, 105-117.

Chen, M., and Y. Zheng, 2004: Vorticity budget investigation of a simulated long-lived mesoscale vortex in South China. $A d v$. Atmos. Sci., 21, 928-940.

Cohen, J., J. A. Screen, J. C. Furtado, M. Barlow, D. Whittleston, D. Coumou, J. Francis, K. Dethloff, D. Entekhabi, J. Overland, and J. Jones, 2014: Recent Arctic amplification and extreme mid-latitude weather. Nature Geosci., 7, 627-637.

Crawford, A. D., and M. C. Serreze, 2016: Does the summer arctic frontal zone influence arctic ocean cyclone activity? J. Climate, 29, 4977-4993.

Grotjahn, R., 1996: Vorticity equation terms for extratropical cyclones. Mon. Wea. Rev., 124, 2843-2858, doi:10.1175/1520-0493(1996) 124<2843:VETFEC $>2.0 . \mathrm{CO} ; 2$.

Hersbach, H., and co-authors, 2020: The ERA5 global reanalysis. Quart. J. Roy. Meteor. Soc., 146, 1999-2049, doi:10.1002/ qj.3803.

Knievel, J., and R. H. Johnson, 2003: A scale-discriminating vorticity budget for a mesoscale vortex in a midlatitude, continental mesoscale convective system. J. Atmos. Sci., 60, 781-794.

Koyama, T., J. Stroeve, J. Cassano, and A. Crawford, 2017: Sea ice loss and arctic cyclone activity from 1979 to 2014. J. Climate, 30, $4735-4754$.

Neu, U., and co-authors, 2013: A community effort to intercompare extratropical cyclone detection and tracking algorithms. Bull. Amer. Meteor. Soc., 94, 529-547.

Raymond, D. J., and C. L. Carrillo, 2011: The vorticity budget of developing typhoon Nuri (2008). Atmos. Chem. Phys., 11, 147163.

Screen, J. A., T. J. Bracegirdle, and I. Simmonds, 2018: Polar climate change as manifest in atmospheric circulation. Current Climate Change Reports, 4, 383-395.

Serreze, M. C., and J. A. Francis, 2006: The Arctic amplification debate. Climatic Change, 76, 241-264.

Simmonds, I., 2000: Size changes over the life of sea level cyclones in the NCEP reanalysis. Mon. Wea. Rev., 128, 4118-4125.

Simmonds, I., and K. Keay, 2009: Extraordinary September arctic sea ice reduction and their relationship with storm behavior over 1979-2008. Geophys. Res. Lett., 36, L19715, doi:10.1029/2009 GL039810.

Simmonds, I., and I. Rudeva, 2012: The great Arctic cyclone of August 2012. Geophys. Res. Lett., 39, L23709, doi:10.1029/ 2012 GL054259.

Simmonds, I., and I. Rudeva, 2014: A comparison of tracking methods for extreme cyclones in the Arctic basin. Tellus A: Dynamic Meteorology and Oceanography, 66, 25252, doi:10.3402/ tellusa.v66.25252.

Shinoda, Y., R. Kawamura, T. Kawano, and H. Hiroyuki, 2020: Dynamical role of the Changbai Mountains and the Korean Peninsula in the wintertime quasi-stationary convergence zone over the Sea of Japan. Int. J. Climatol., 2020, 1-14.

Tanaka, H. L., A. Yamagami, and S. Takahashi, 2012: The structure and behavior of the arctic cyclone in summer analyzed by the JRA-25/JCDAS data. Polar Sci., 6, 55-69.

Yamagami, A., M. Matsueda, and H. L. Tanaka, 2017: Extreme Arctic cyclone in August 2016. Atmos. Sci. Let., 18, 307-314.

Yamagami, A., M. Matsueda, and H. L. Tanaka, 2018: Mediumrange forecast skill for extraordinary Arctic cyclones in summer of 2008-2016. Geophys. Res. Lett., 45, 4429-4437.

Yamagami, A., M. Matsueda, and H. L. Tanaka, 2019: Skill of medium-range reforecast for summertime extraordinary Arctic Cyclones in 1986-2016. Polar. Sci., 20, 107-116.

Zhang, J., 2005: Warming of the arctic ice-ocean system is faster than the global average since the 1960s. Geophys. Res. Lett., 32, L19602, doi:10.1029/2005GL024216.

Manuscript received 8 January 2021, accepted 6 April 2021

SOLA: https://www.jstage.jst.go.jp/browse/sola/ 